\title{
A Method to Control Dynamic Errors of the Stylus-Based Probing System for the Surface Form Measurement of Microstructures
}

\author{
Hui Fang, Bin Xu, Deqiang Yin, and Shiping Zhao \\ School of Manufacturing Science and Engineering, Sichuan University, 24 South No. 1 Ring Road, Chengdu 610065, China
}

Correspondence should be addressed to Bin Xu; bin_xu@outlook.com and Deqiang Yin; deqiang.yin@scu.edu.cn

Received 25 December 2015; Accepted 12 April 2016

Academic Editor: James Piao

Copyright (C) 2016 Hui Fang et al. This is an open access article distributed under the Creative Commons Attribution License, which permits unrestricted use, distribution, and reproduction in any medium, provided the original work is properly cited.

\begin{abstract}
Dynamic errors of the stylus-based probing system seriously affect the measurement accuracy in surface quality detection on microstructures. A method, which is focused on measuring the surface form of microgears, microholes on the injector nozzle, and so forth, is proposed to control dynamic errors of the stylus-based probing system. Firstly, two kinematical models were built to systematically investigate the dynamic performance of the stylus-based probing system, and the relationship between the measuring force, scanning speed, fidelity, and dynamic errors was described. Then, numerical simulations of the surface form measurement, in which a sine-shaped microstructure was taken as a target surface, were carried out. Results of the numerical simulations reveal that the dynamic errors were affected by the scanning speed and the initial position of the measured specimen. Lastly, a method to control dynamic errors by properly setting up the scanning speed and the initial position of the specimen was proposed for the surface form measurement of microstructures.
\end{abstract}

\section{Introduction}

Microstructures, such as microgears and microholes, are widely used in precision industries [1-3]. The working performance of microstructure-based systems and devices is primarily determined by the geometric accuracy of the employed microstructures [4]. It is, therefore, important to carry out high-accuracy surface form measurement of microstructures so that the form accuracy of microstructures can be improved and guaranteed in the manufacturing process. However, due to the fact that many of the microstructures have the characteristics of high-aspect-rations and steep surface slopes, it is difficult to carry out high-accuracy surface form measurement on microstructures [5-7].

The stylus-based probing system is an important technique for measuring the surface form of microstructures because of the advantages of convenient utilization, insensitivity to surface contaminants, and high measurement accuracy [8-10]. In the authors' previous study, two types of stylusbased profiling systems, one of which has an elastic element and the other one without it, were used to measure the surface form of gear-shaped microstructures [2, 11]. Measurement results show that it had an obvious form distortion on the top of the measured microstructures and the measurement repeatability got worse on the side flanks. The stylus-based probing system employs a stylus to trace the surface of the target surface, and the spatial position of the stylus tip is recorded and employed to reconstruct the surface form of the target surface. However, the trace of the stylus tip cannot always follow the surface variation of the measurement target, because the motion of the stylus tip is affected by the kinetics of the stylus-based probing system. For instance, the stylus tip separates from the measured surface during scanning process, which is referred to as stylus flight phenomenon.

Over the years, work has been conducted to resolve the issue of stylus flight. Damir proposed a method to choose the suitable spring stiffness of a stylus-based profiling system [12]. Liu et al. developed an active damping control method to modify a stylus instrument and confirmed that the stylus instrument could get better working performance while the damping factor was in the region between 0.5 and 0.7 [8], which meets the research results of Whitehouse [13, 14]. 
McCool pointed out that the reconstructed profile deduced from the stylus profile traces was distorted by the nonlinear filtering effect of the finite stylus tip and by the failure at high enough tracing speeds of the stylus to maintain contact with the profile being traced [15]. Pawlus et al. discussed the influence of the stylus flight on change of surface topography parameters $[10,16]$. Liu and Tian et al. built dynamic models, which took the Hertzian contact and nonlinear damping forces into consideration, to analyse the stylus flight phenomenon $[9,17,18]$. The above approaches focused on the modification of hardware of the stylus instrument, such as modifications of the damping factor, spring stiffness, and the mass of a stylus. The modification of hardware would cause high costs in economy and technology. Therefore, a simple and cost-effective method to depress the stylus flight is desired in precision engineering.

This paper presents a method to control dynamic errors of the stylus-based probing system for the surface form measurement of microstructures. Firstly, two kinematical models were built to systematically investigate the dynamic performance of the stylus-based probing system, and relevant theoretical work was given in detail. Then, numerical simulations of the surface form measurement were carried out while taking a sine-shaped microstructure as a target surface. Lastly, a method was proposed to control the dynamic errors on the surface form measurement of microstructures.

\section{Kinematical Model of the Stylus-Based Probing System}

The stylus-based probing system employs a stylus to traverse the target surface. The vertical position of the stylus changes following the variation of the target surface, and it is measured by a displacement sensor. The output of the displacement sensor, the position variation of the stylus in vertical direction, is employed to reconstruct the surface form for the measurement specimen. However, the vertical position of the stylus cannot always exactly follow the variation of the measured surface in the region with steep slopes and huge slope variations, which results in form distortion and is known as the stylus flight phenomenon as shown in Figure 1(a).

Typical stylus-based probing systems have the types of lever mechanism, flexure hinge mechanism, and the airbaring mechanism according to how the stylus is supported $[2,9]$ and can be generally modelled by a second-order kinematical system as shown in Figure 1(b). In this kinematical system, the vertical position of the stylus is denoted by $y$, which is subjected to the measured surface form $y_{s}$ and crucial parameters of the stylus-based probing system. Neglecting the friction force between the stylus tip and the measured surface for clarity, the equation of the stylus motion can be expressed as follows:

$$
-m g-m \ddot{y}-k y-c \dot{y}+F=0,
$$

where $m$ is the equivalent mass of the stylus-based probing system, $g$ is the gravitational acceleration, $k$ is the spring stiffness, $c$ is the damping coefficient, $\dot{y}$ and $\ddot{y}$ are the velocity and acceleration of the stylus motion along the $Y$-direction, and $F$ is the measuring force, namely, the interaction force between the stylus tip and the measured surface. The surface scanning is carried out along the $X$-direction with a speed $v$. The $Y$-direction is aligned to the direction of the gravity, and the origin $O$ of $Y$ coordinate is the position of the stylus tip while the spring is not stretched. The position $A$ is assumed to be the position where the spring is stretched by its gravity and the zero point of the probe sensing. The distance of the average height of the measured surface to the zero point of the probe sensing, position $A$, is defined to be the initial position of the measured surface.

In the surface scanning process, the stylus tip always contacts with the measured surface while the stylus flight phenomenon does not occur. Under this condition, the measuring force should be greater than zero. Therefore, the measuring force $F$ is an important parameter to represent the dynamic behavior of the stylus-based probing system. Then, (1) is rearranged as

$$
F=m \ddot{y}+c \dot{y}+k y+m g .
$$

In the case that the stylus flight phenomenon does not occur, which means that the stylus tip is contacting with the measured surface, the stylus motion follows the variation of the measured surface. Therefore, the velocity and the acceleration of the stylus motion are equal to those of the variation of the measured surface, which are expressed by the following equation:

$$
\begin{aligned}
& \dot{y}=\dot{y}_{s} \\
& \ddot{y}=\ddot{y}_{s},
\end{aligned}
$$

where $y_{s}$ is the measured surface. Assuming the measured surface is of a form of $y_{s}=f(x)$, (3) becomes

$$
\begin{aligned}
& \dot{y}=\dot{x} f^{\prime}(x) \\
& \ddot{y}=(\dot{x})^{2} f^{\prime \prime}(x),
\end{aligned}
$$

where $\dot{x}$ is equal to the scanning speed $v, f^{\prime}(x)$ is the slope $\rho$ of the measured surface, and $f^{\prime \prime}(x)$ is the variation of the surface slope and is denoted by $\dot{\rho}$. Therefore, the measuring force expressed by (2) is rewritten as

$$
F=m v^{2} \dot{\rho}+c v \rho+k f(x)+m g .
$$

While the stylus tip contacts with the measured surface, $F$ should be greater than zero, which is expressed as

$$
F=v^{2} \dot{\rho}+2 \xi \omega v \rho+\omega f(x)+g>0,
$$

where $\xi=c / 2 \sqrt{\mathrm{km}}$ is the damping ration and $\omega=\sqrt{\mathrm{k} / \mathrm{m}}$ is the resonant frequency of the stylus-based probing system. In order to guarantee that the measuring force is greater than zero, the general solutions are to improve the resonant frequency $\omega$, adjust the damping ration $\xi$, and reduce the scanning speed $v$.

The methods to improve the resonant frequency $\omega$ are the increase of spring stiffness $k$ and the reduction of equivalent 


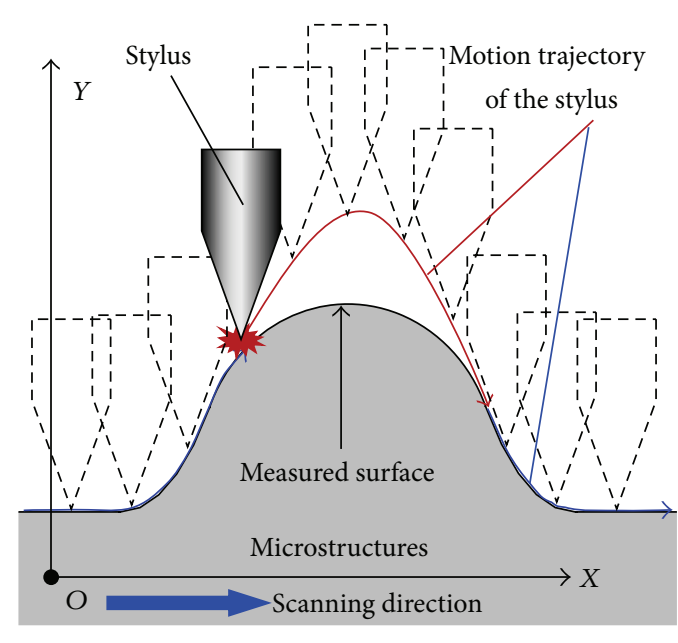

(a)

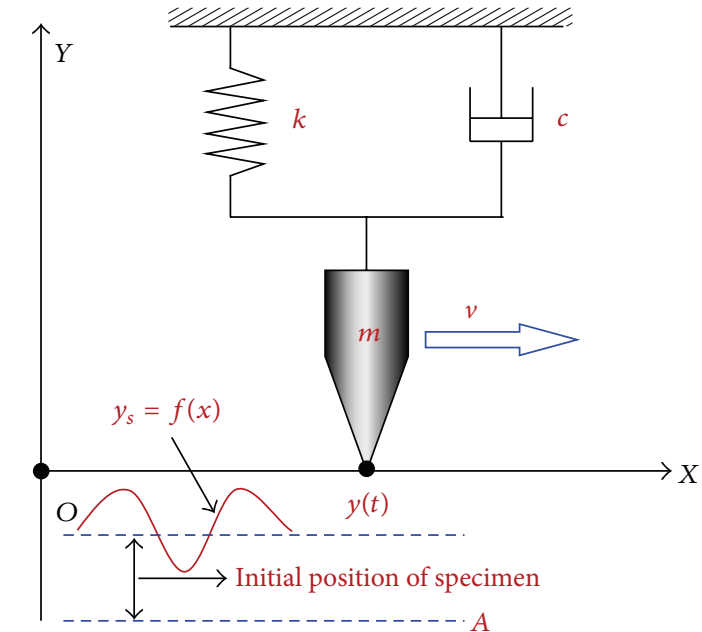

(b)

Figure 1: (a) Schematic of the stylus flight. (b) Kinematical model of the stylus-based probing system.

mass $m$. The increase of the spring stiffness $k$ would increase the measuring force $F$, which possibly results in the damage of the measured surface. On the other hand, the equivalent mass $m$ also cannot be reduced without limitation, because the geometric parameters of the stylus should meet the requirement of functional requirements. As shown in (6), the effect on the measuring force $F$ is relevant to the surface slope $\rho$. If the surface slope $\rho$ is positive, it is better that the damping ration $\xi$ be greater; otherwise, it should be smaller. It should be mentioned that the slope $\rho$ of the measured surface has the potential to be distributed from negative to positive. Therefore, it is hard to realize an appropriate adjustment of the damping ration $\xi$, or the appropriate adjustment would result in the increase of costs in economy and technology. The effect of the scanning speed $v$ to the measuring force $F$ is the function of the surface slope $\rho$ and its variation $\dot{\rho}$. Generally, it is believed that a better measurement result can be obtained at a slower scanning speed. However, the slower scanning speed would lead to the reduction of work efficiency.

In this case the stylus flight phenomenon occurs, which means the stylus tip is out of contact with the measured surface. In this condition, the measuring force is reduced to zero and the stylus motion is free oscillation. Figure 2 illuminates the kinematical model of the stylus flight. Assuming that the stylus separates from the measured surface on the point $P_{0}\left(x_{P_{0}}, y_{P_{0}}\right)$ with a vertical speed $v_{P_{0}}$, the time on this moment is set to zero for clarity. The vertical position $y_{P_{t}}$ of the stylus is a time-varying quantity and it is the solution of (7), which is derived from (2). Consider

$$
\ddot{y}+\frac{c}{m} \dot{y}+\frac{k}{m} y=-g .
$$

Equation (7) is linear and of second order with a constant coefficient. The general solution is

$$
y=e^{\alpha t}\left[C_{1} \cos \beta t+C_{2} \sin \beta t\right],
$$

where $\alpha=-c / 2 m$ and $\beta=\sqrt{4 k m-c^{2}} / 2 m$. To (8), the characteristic equation is expressed as

$$
r^{2}+\frac{c^{2}}{m^{2}} r+\frac{k}{m}=0
$$

Due to $c<2 \sqrt{\mathrm{km}}$, the solution to this characteristic equation is

$$
r_{1,2}=-\frac{c}{2 m} \pm i * \frac{\sqrt{4 k m-c^{2}}}{2 m} .
$$

To (8), the general solution can be expressed as follows:

$$
\begin{aligned}
y_{1} & =e^{-(c / 2 m) t}\left[C_{1} \cos \frac{\sqrt{4 k m-c^{2}}}{2 m} t\right. \\
& \left.+C_{2} \sin \frac{\sqrt{4 k m-c^{2}}}{2 m} t\right]
\end{aligned}
$$

To (8), the particular solution is

$$
y_{2}=-\frac{m g}{k} .
$$

The solution to (8) can be derived from (11) and (12) and expressed as follows:

$$
\begin{aligned}
y & =e^{-(c / 2 m) t}\left[C_{1} \cos \frac{\sqrt{4 k m-c^{2}}}{2 m} t\right. \\
& \left.+C_{2} \sin \frac{\sqrt{4 k m-c^{2}}}{2 m} t\right]-\frac{m g}{k} .
\end{aligned}
$$

The initial condition of (13) is that $y=y_{P_{0}}$ and $d y / d t=$ $v_{P_{0}}$ while $t=0$. Substituting this initial condition into (13), the parameters $C_{1}$ and $C_{2}$ can be found. Consider

$$
\begin{aligned}
& C_{1}=\frac{m g}{k}+y_{P_{0}} \\
& C_{2}=\frac{2 m v_{-P_{0}}+m g c / k+c y_{P_{0}}}{\sqrt{4 k m-c^{2}}} .
\end{aligned}
$$




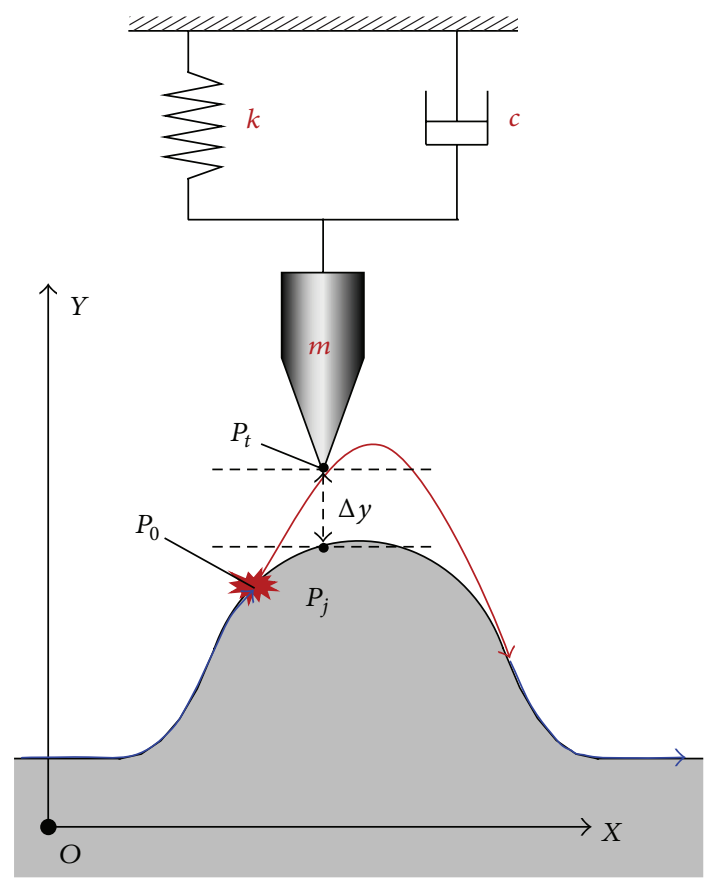

FIGURE 2: Kinematical model of the stylus flight.

Then, (13) can be rewritten as

$$
\begin{aligned}
y= & e^{-(c / 2 m) t}\left[\left(\frac{m g}{k}+y_{P_{0}}\right) \times \cos \frac{\sqrt{4 k m-c^{2}}}{2 m} t\right. \\
& \left.+\left(\frac{2 m v_{-P_{0}}+m g c / k+c y_{P_{0}}}{\sqrt{4 k m-c^{2}}}\right) \sin \frac{\sqrt{4 k m-c^{2}}}{2 m} t\right] \\
& -\frac{m g}{k} .
\end{aligned}
$$

Equation (15) gives out the motion of the stylus while the stylus flight occurs.

\section{Numerical Simulation and Analysis of Dynamic Performance}

3.1. Effect of Measurement Setup on Dynamic Performance. The parameters of the stylus-based probing system and the measurement setup affect the dynamic performance (e.g., fidelity, measuring force, and dynamic errors), which were analysed by numerical simulations based on the kinematical model expressed by (1). In the numerical simulations, the parameters of a practical stylus-based probing system were employed and given as $m=3.5 \mathrm{~g}, k=2.7 \mathrm{~N} / \mathrm{m}$, and $c=$ $0.00144 \mathrm{Nsm}^{-1}$ [19]. A sine-shaped microstructure expressed by (16) with amplitude of $200 \mu \mathrm{m}$ and period of $100 \mu \mathrm{m}$ was used as the measurement specimen. The interval of the measured surface discretization was set to $0.1 \mu \mathrm{m}$, and the scanning interval was set to $1 \mu \mathrm{m}$. Consider

$$
y_{s}=200 \times \cos \left(\frac{x \times \pi}{50}\right)-b
$$

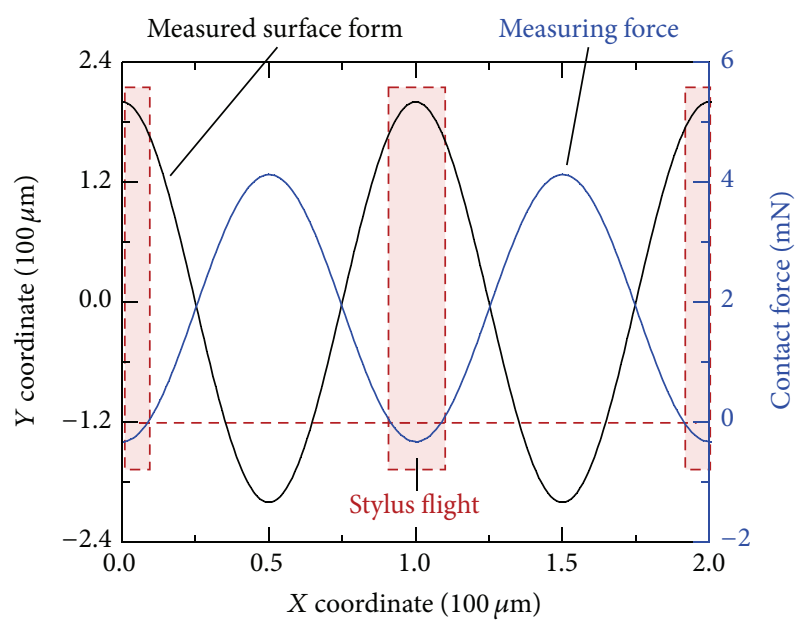

FIgURE 3: Measured surface form and the measuring force.

in which the unit is micrometer and the parameter $b$ is used to simulate the initial setup of the specimen's position. The stylus tip is stretched $12.7 \mathrm{~mm}$ below the $x$-axis by its gravity, where it was assumed to be the zero point of the displacement sensing of the stylus-based probing system. If the parameter $b$ is $12 \times 10^{3}$, the distance of the measured form to the zero point of the displacement sensing is $0.7 \mathrm{~mm}$.

Firstly, the fidelity of form measurement, which means how much of the measured surface could be exactly measured without the stylus flight phenomenon, was investigated by the numerical simulation. The scanning speed $c$ was set to $1 \mathrm{~mm} / \mathrm{s}$ and the initial position of the measured surface was set to $0.7 \mathrm{~mm}$. The kinematical model expressed by (6) about the measuring force was utilized to detect whether the stylus flight phenomenon occurred or not. Figure 3 shows the simulation results. The curve of the measuring force, which is plotted by the raw output of (6), presents a sine shape and has a phase difference of $\pi$ to the measured surface form. The values of the measuring force distribute from $-0.32 \mathrm{mN}$ to $4.12 \mathrm{mN}$. In practice, the measuring force cannot be smaller than zero. The region marked by red blocks, where the measuring force is negative, indicates that the stylus flight occurred in the region near the peak of the measured form. In this case, $82.79 \%$ of the measured surface can be exactly measured.

The influence of the surface geometrical characteristic to the stylus flight was analysed in Figure 4. In this figure, the surface slope and the rate of slope variation were drawn according to their $X$ coordinates, and the region of the stylus flight was covered by red blocks. It can be found that the rate of slope variation has a strong impact on the stylus flight compared to the surface slope, which means that the stylus tip usually lost contact in the region with a huge slope variation.

The effect of the scanning speed on the measuring force and the fidelity was investigated by changing the speed from $0.01 \mathrm{~mm} / \mathrm{s}$ to $4 \mathrm{~mm} / \mathrm{s}$, of which the result was shown in Figure 5. In this figure, the black curve is plotted by the maximum value of the measuring force at each scanning speed, and the red one is plotted by the fidelity at each 


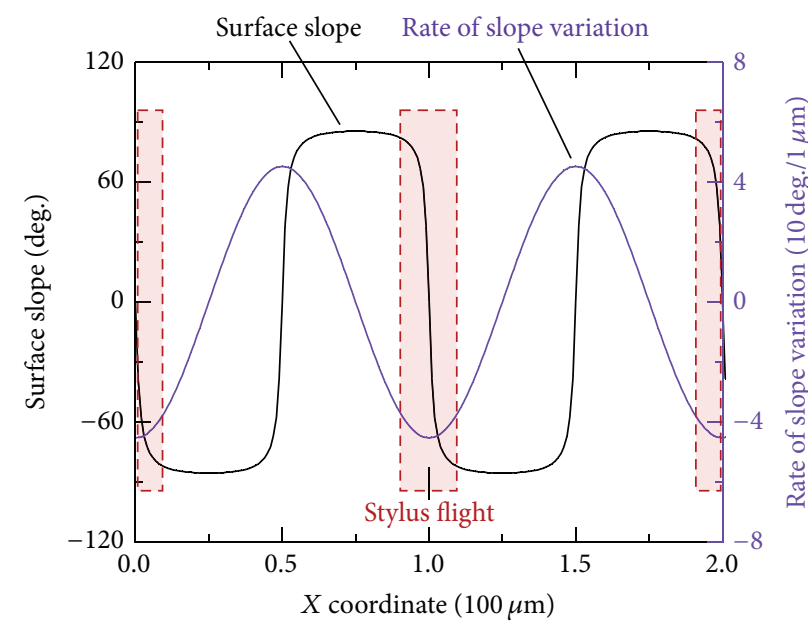

FigURE 4: Effect of the surface slope and rate of slope variation on stylus flight.

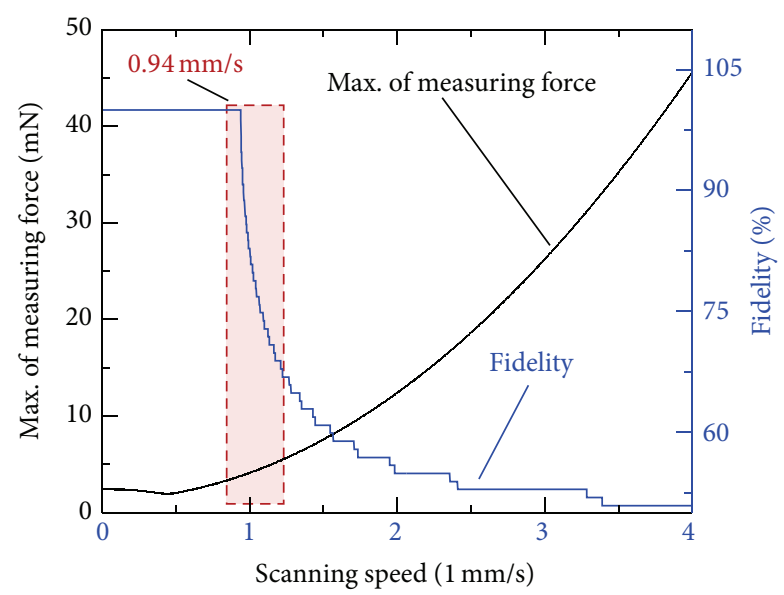

FIGURE 5: Effect of the scanning speed on dynamic performance.

scanning speed. The results show that while the scanning speed exceeded $0.94 \mathrm{~mm} / \mathrm{s}$, the fidelity would be rapidly falling and the measuring force would be rising up very fast.

The initial position of the measured specimen is another crucial factor to affect the dynamic performance. Therefore, the distance of the measured specimen to the zero point of displacement sensing was set from $0.2 \mathrm{~mm}$ to $0.7 \mathrm{~mm}$ and was used as a variable in the numerical simulation. The relation of the specimen's initial position with the measuring force and the fidelity is shown in Figure 6. As shown in the figure, while the initial position is increasing, both the measuring force and the fidelity are increasing. However, it should be known that the measuring force is generally expected to be in the region of few micronewtons.

3.2. Effect of Measurement Setup on Dynamic Error. Higher scanning speed and lower measuring force are desired in practical measurement tasks. However, both the increase of scanning speed and the reduction of the measuring force could potentially result in stylus flight, which would

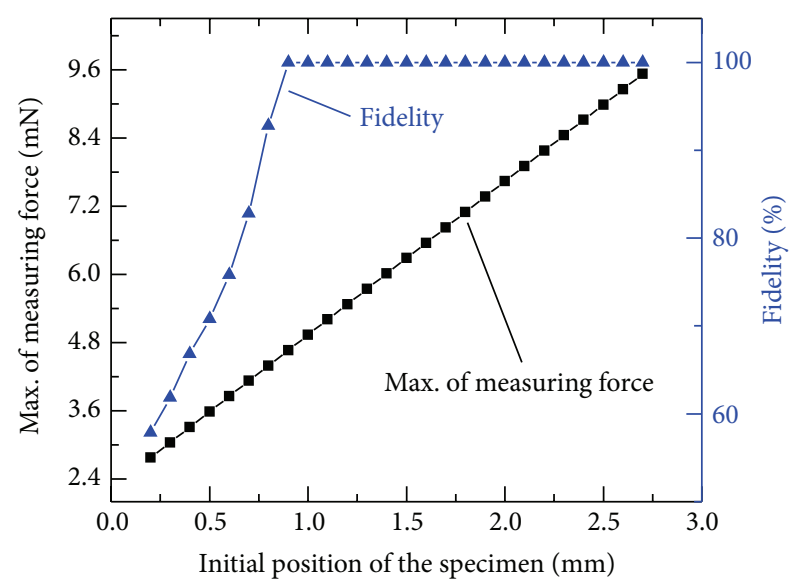

Figure 6: Effect of the initial position of the specimen on dynamic performance.

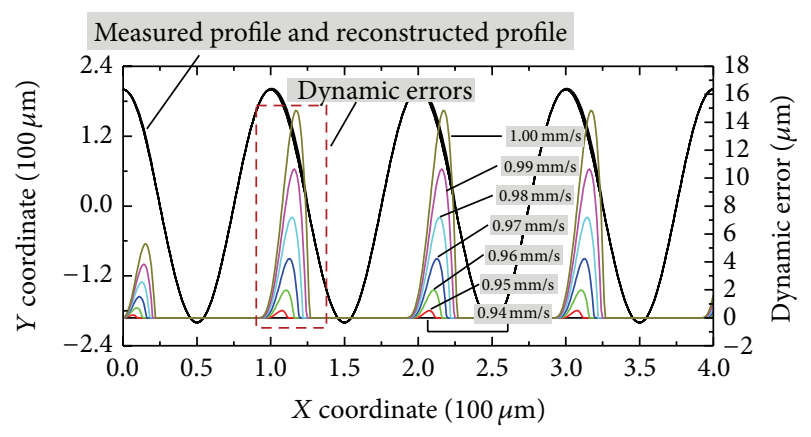

Figure 7: Effect of scanning speed $(0.94 \mathrm{~mm} / \mathrm{s}$ to $1 \mathrm{~mm} / \mathrm{s})$ on dynamic errors.

introduce dynamic errors into measurement results. The above numerical simulation method was also used to analyse the effect of measurement setup on dynamic errors. There is only one difference, which is that the motion trace of stylus tip was computed by (15), and the initial condition was obtained from (5). The motion trace of the stylus tip in whole scanning process was the measurement results, which was used to reconstruct the surface form for the measured profile. The difference of the reconstructed profile and the measured profile was seen as the dynamic errors introduced by the stylus flight.

Figure 5 indicates that while the scanning speed exceeded $0.94 \mathrm{~mm} / \mathrm{s}$, the stylus flight would occur. Therefore, the scanning speed was set from $0.94 \mathrm{~mm} / \mathrm{s}$ to $1.00 \mathrm{~mm} / \mathrm{s}$ with an interval of $0.01 \mathrm{~mm} / \mathrm{s}$, and the initial position of the measured specimen was set to $0.7 \mathrm{~mm}$. The results of the numerical simulation are shown in Figure 7. The reconstructed profiles at each scanning speed and the measured profile are covered with each other due to their small differences. The dynamic errors increase from smaller than $1 \mu \mathrm{m}$ to about $15 \mu \mathrm{m}$. The increase ratio is approximately $3 \mu \mathrm{m}$ per $0.01 \mathrm{~mm} / \mathrm{s}$. The region marked by a red block in this figure indicates that the position with the maximum dynamic errors is not exactly on the peaks of the measured microstructures. In this case, the position with the maximum dynamic errors slightly passed 


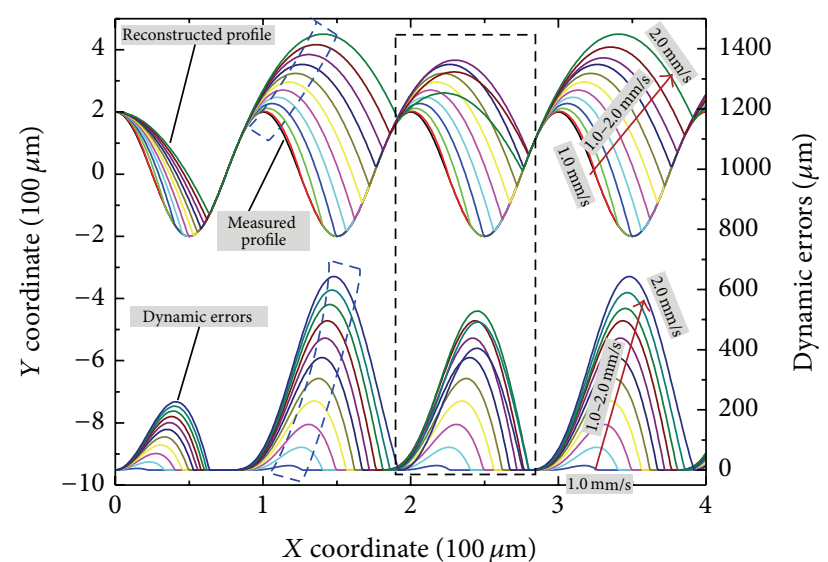

FIGURE 8: Effect of scanning speed ( $1 \mathrm{~mm} / \mathrm{s}$ to $2 \mathrm{~mm} / \mathrm{s}$ ) on dynamic errors.

over the peaks. The other feature that should be mentioned is that the dynamic errors present periodic features expect in the first period of the measured surface. This is because the initial condition of the stylus flight is different; namely, the separation position and the vertical speed of stylus tip differ.

The effect of the scanning speed on the dynamic errors was studied on a larger scale, $1.00 \mathrm{~mm} / \mathrm{s}$ to $2.00 \mathrm{~mm} / \mathrm{s}$, and the results are shown in Figure 8. The dynamic errors sharply increased from $15 \mu \mathrm{m}$ to about $650 \mu \mathrm{m}$, which would be out of the acceptable error range. The feature of the dynamic errors on the third period of the measured surface does not completely repeat with it on the second period. This is because the separation position of the stylus tip changed after a serious stylus flight. But this feature is repeated over two periods of the measured profile.

The initial position of the measured specimen is also very important and seriously affects the dynamic errors. In the numerical simulation, the initial position of the measured specimen was set from $0.1 \mathrm{~mm}$ to $0.6 \mathrm{~mm}$ while the scanning speed remained $1 \mathrm{~mm} / \mathrm{s}$. The measurement results and corresponding dynamic errors at different initial position are shown in Figure 9. While the initial position was increasing, the dynamic errors obviously reduced from about $250 \mu \mathrm{m}$ to smaller than $1 \mu \mathrm{m}$. Comparing the results shown in Figures 7-9, the curve shape of dynamic errors presents a subtle asymmetry that the dynamic errors on the flank side against the stylus scanning motion are obviously greater than on another side.

\section{Control Method of Dynamic Errors}

The above numerical simulation and analysis reveals that the measurement setup (i.e., scanning speed and the initial position of the specimen) should be properly chosen. Otherwise, excessive measuring force would make the stylus damage the measured surface, and unacceptable dynamic errors would reduce the reliability of the measurement results. The method to control the dynamic errors into the acceptable range was proposed based on the prediction of dynamic errors and the measuring force. Firstly, the surface scanning is simulated

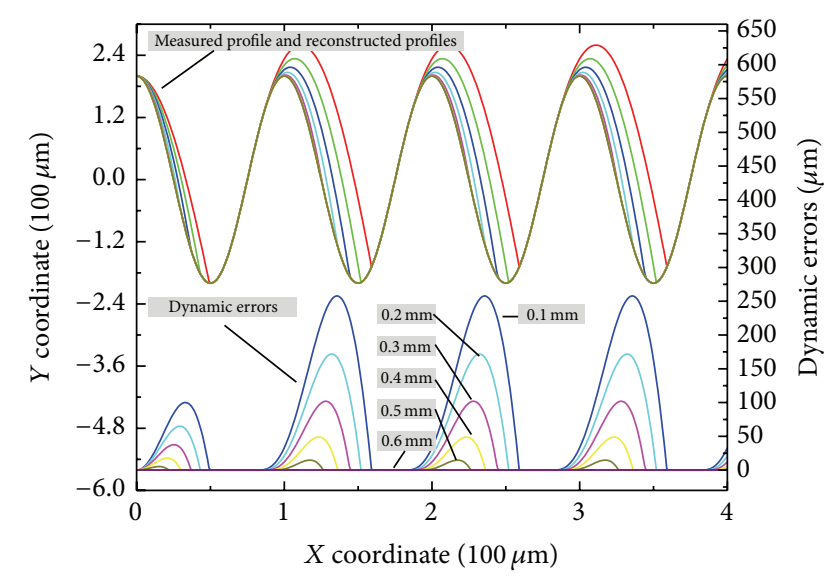

FIgURE 9: Effect of initial position on dynamic errors.

by the method introduced in Section 3.2, and the measuring force is analysed by the method introduced in Section 3.1. The maximum values of the dynamic errors and the measuring force are recorded as a set of data. Then change the values of the scanning speed and the initial position of the specimen; step 1 is repeated and more sets of data are acquired. All the obtained sets of data present the relationship graph between the dynamic performance and the measurement setup. Lastly, the optimized selections of the scanning speed and the initial position of the specimen can be carried out from the relationship graph according to the requirements about the dynamic errors and the measuring force of a measurement task.

The sine-shaped microstructure with steep slopes expressed by (16) was employed by the numerical simulation to verify the proposed method. The scanning speed was set from $0.93 \mathrm{~mm} / \mathrm{s}$ to $2.00 \mathrm{~mm} / \mathrm{s}$, and the initial position of the specimen was set from $0.3 \mathrm{~mm}$ to $0.9 \mathrm{~mm}$. The relationship graph between the dynamic performance and the measurement setup was plotted in Figure 10. Assume that the measurement task requires that the dynamic errors should be smaller than $10 \mu \mathrm{m}$ and the measuring force should be smaller than $5 \mathrm{mN}$. Then, Figure 10 was reprocessed with a limitation of the $10 \mu \mathrm{m}$ dynamic errors and was shown in Figure 11. The scale of the colour bar was redefined to easily get more details of the measuring force. The regions of the scanning speed and the initial position of the specimen would be the choice which would make the measurement result meet the requirements of the measurement task. In addition, the best measurement setup would be $1.1 \mathrm{~mm} / \mathrm{s}$ scanning speed and $0.9 \mathrm{~mm}$ initial position for higher measurement efficiency.

\section{Conclusion}

In order to enhance the form measurement accuracy of microstructures such as microgears and microholes on the injector nozzle, research on the dynamic feature of stylusbased probing systems has been carried out in this paper. Results of numerical simulations reveal that the rate of the 


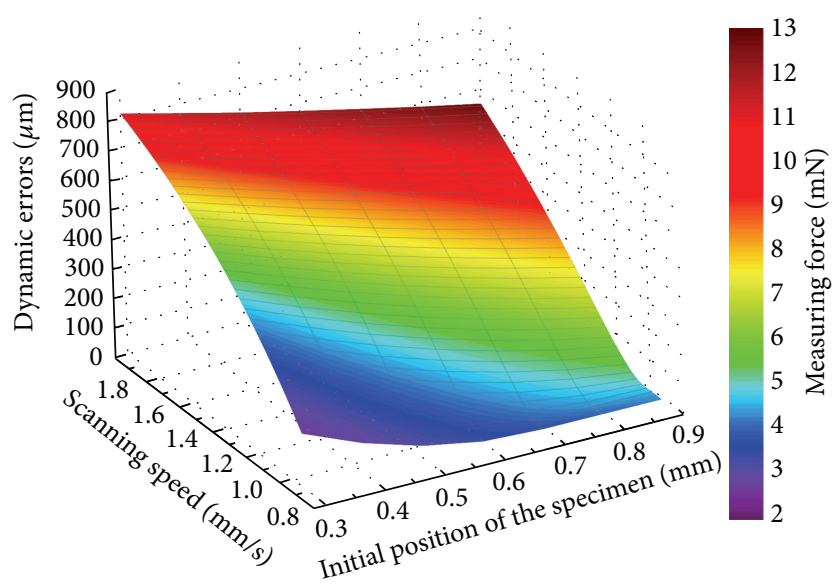

FIGURE 10: Relationship graph between the dynamic performance and the measurement setup.

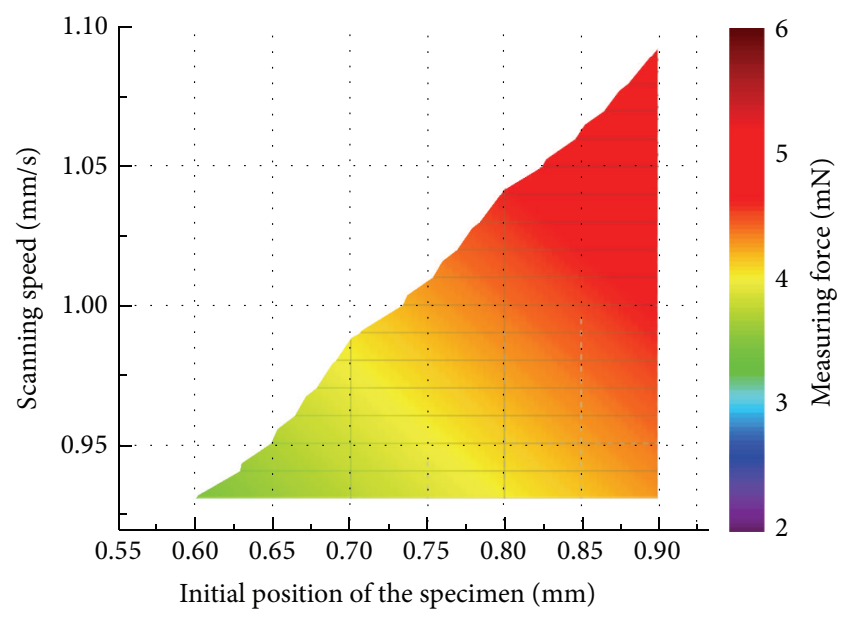

FIgURE 11: Optimized selections of the measurement setup.

slope variation of the measured surface greatly affects the dynamic performance of the stylus-based probing system. Furthermore, it is hard to control the dynamic performance by decreasing scanning speed and increasing spring stiffness due to requirements on measurement efficiency and measuring force. Afterwards, a simple and cost-effective method to control the dynamic performance has been proposed without changing the hardware of the stylus-based probing system. This method employs kinematical models to predict the influence of the measurement setup on dynamic performance, and then the optimal scanning speed and the initial position of the measured sample can be selected according to requirements on measurement efficiency and measuring force.

It should be noted that the proposed method did not take adhesion, friction, and elastic deformation into consideration and the constructed dynamic model is two-dimensional. These issues remain to be investigated by future work.

\section{Competing Interests}

The authors declare that they have no competing interests.

\section{Acknowledgments}

This work is supported by the National Natural Science Foundation of China (Grant nos. 51405314, 11302141, and 61006403), by the Fundamental Research Funds for the Central Universities (Grant no. 2015SCU04A12), and by the LPMT, CAEP (Grant no. KF15004).

\section{References}

[1] H. N. Hansen, K. Carneiro, H. Haitjema, and L. De Chiffre, "Dimensional micro and nano metrology," CIRP AnnalsManufacturing Technology, vol. 55, no. 2, pp. 721-743, 2006.

[2] E. Peiner, M. Balke, and L. Doering, "Form measurement inside fuel injector nozzle spray holes," Microelectronic Engineering, vol. 86, no. 4-6, pp. 984-986, 2009.

[3] B. Xu, Y. Shimizu, T. Takeishi, S. Ito, and W. Gao, "Surface form measurement and analysis of a cylindrical workpiece with microstructures," Journal of Advanced Mechanical Design, Systems and Manufacturing, vol. 6, no. 6, pp. 936-948, 2012.

[4] B.-F. Ju, Y.-L. Chen, W. Zhang, and F. Z. Fang, "Rapid measurement of a high step microstructure with $90^{\circ}$ steep sidewall," Review of Scientific Instruments, vol. 83, no. 1, Article ID 013706, 2012.

[5] M. B. Bauza, S. C. Woody, B. A. Woody, and S. T. Smith, "Surface profilometry of high aspect ratio features," Wear, vol. 271, no. 34, pp. 519-522, 2011.

[6] J. D. Claverley and R. K. Leach, "A vibrating micro-scale CMM probe for measuring high aspect ratio structures," Microsystem Technologies, vol. 16, no. 8-9, pp. 1507-1512, 2010.

[7] H. Fang, B. Xu, W. Chen, H. Tang, and S. Zhao, "A slopeadapted sample-tilting method for profile measurement of microstructures with steep surfaces," Journal of Nanomaterials, vol. 2015, Article ID 253062, 8 pages, 2015.

[8] X. Liu, D. G. Chetwynd, S. T. Smith, and W. Wang, "Improvement of the fidelity of surface measurement by active damping control," Measurement Science \& Technology, vol. 4, no. 12, pp. 1330-1340, 1993.

[9] Y. Tian, X. Liu, D. G. Chetwynd, V. Eichhorn, S. Fatikow, and D. Zhang, "Nonlinear viscoelastic modelling of stylus probing for surface metrology," Precision Engineering, vol. 37, no. 3, pp. 711-720, 2013.

[10] P. Pawlus and M. Śmieszek, "The influence of stylus flight on change of surface topography parameters," Precision Engineering, vol. 29, no. 3, pp. 272-280, 2005.

[11] B. Xu, Y. Shimizu, S. Ito, and W. Gao, "Surface profile measurement of internal micro-structures," International Journal of Precision Engineering and Manufacturing, vol. 14, no. 9, pp. 1535-1541, 2013.

[12] M. N. H. Damir, "Error in measurement due to stylus kinematics," Wear, vol. 26, no. 2, pp. 219-227, 1973.

[13] D. J. Whitehouse, "Dynamic aspects of scanning surface instruments and microscopes," Nanotechnology, vol. 1, no. 1, pp. 93102,1990

[14] D. J. Whitehouse, "Enhancement of instrument and machine capabilities," Nanotechnology, vol. 7, no. 1, pp. 47-51, 1996.

[15] J. I. McCool, "Assessing the effect of stylus tip radius and flight on surface topography measurements," Journal of Tribology, Transactions of the ASME, vol. 106, no. 2, pp. 202-209, 1984.

[16] J. F. Song and T. V. Vorburger, "Stylus flight in surface profiling," Journal of Manufacturing Science and Engineering, vol. 118, p. 11, 1996. 
[17] Y. Tian, X. Liu, D. G. Chetwynd, B. Shirinzadeh, and D. Zhang, "Vibration analysis of stylus instrument for random surface measurement," Precision Engineering, vol. 34, no. 3, pp. 586-591, 2010.

[18] Y. Tian, X. Liu, D. Zhang, and D. G. Chetwynd, "Dynamic modelling of the fidelity of random surface measurement by the stylus method," Wear, vol. 266, no. 5-6, pp. 555-559, 2009.

[19] K.-W. Lee, Y.-J. Noh, W. Gao et al., "Experimental investigation of an air-bearing displacement sensor for on-machine surface form measurement of micro-structures," International Journal of Precision Engineering and Manufacturing, vol. 12, no. 4, pp. 671-678, 2011. 

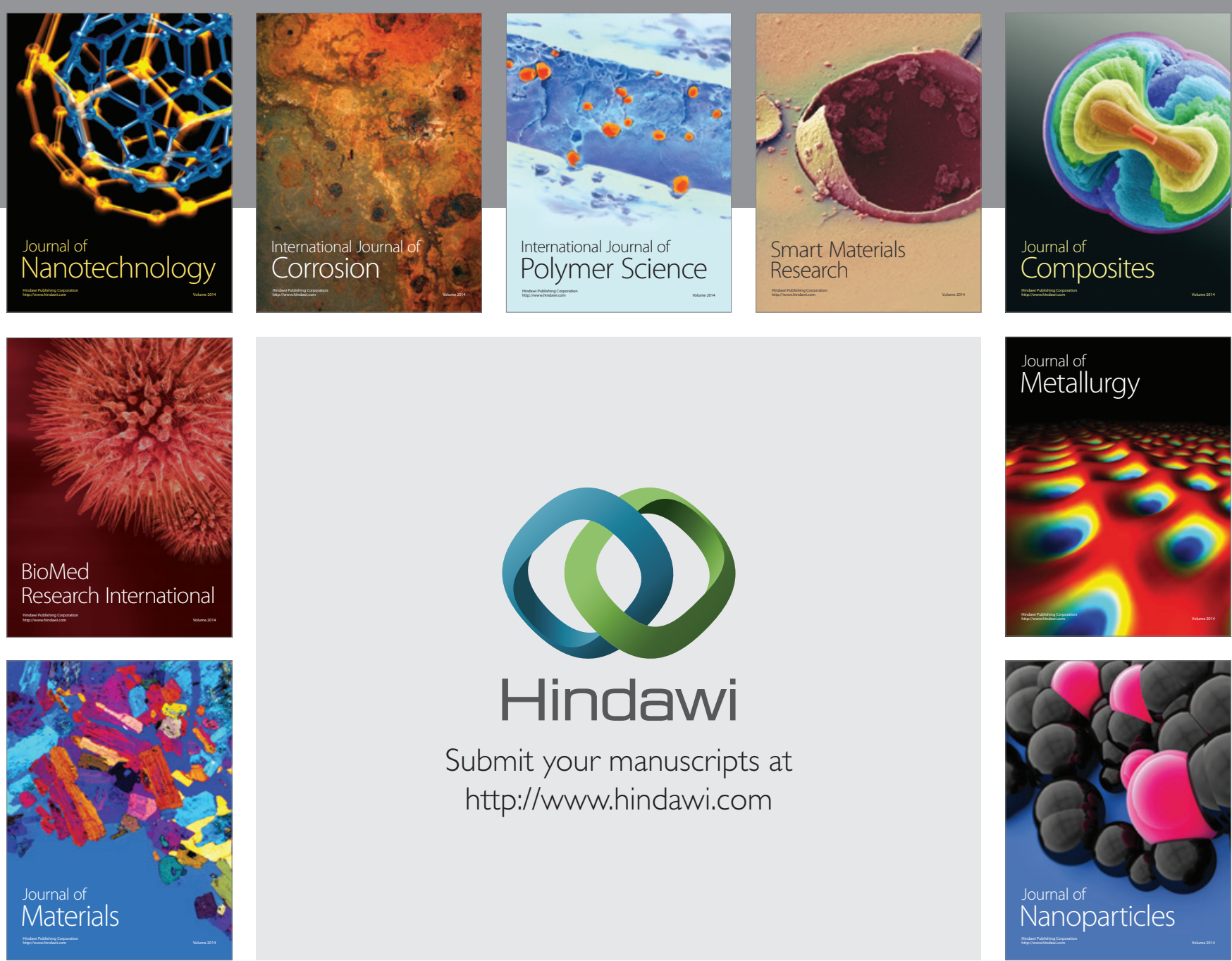

\section{Hindawi}

Submit your manuscripts at

http://www.hindawi.com

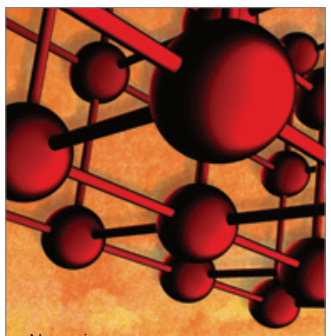

Materials Science and Engineering
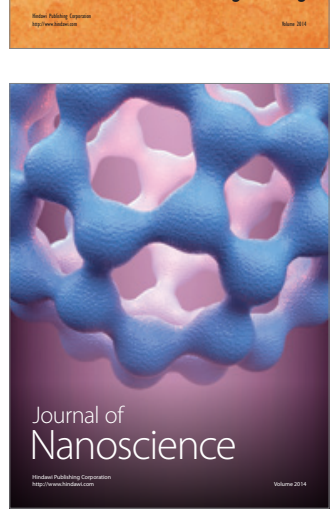
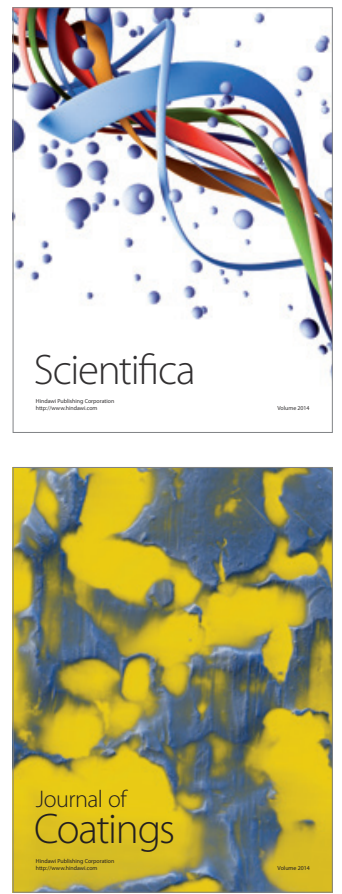
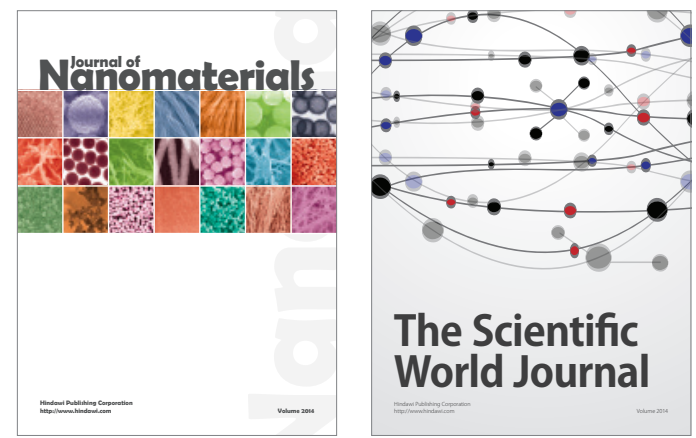

The Scientific World Journal
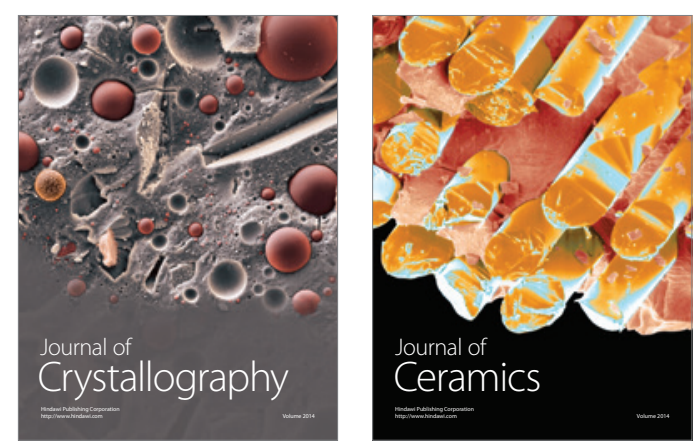
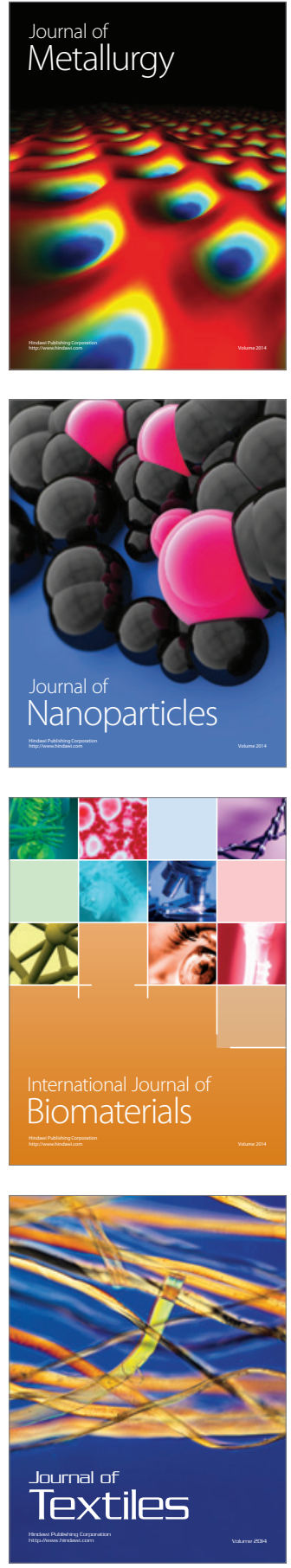